\title{
PRINCIPIOS, APLICACIONES Y RETOS DEL APRENDIZAJE CONECTADO
}

\author{
Moisés Esteban-Guitart \\ University of Girona (Spain) \\ Daniela K. DiGiacomo \\ University of Kentucky (USA) \\ William R. Penuel \\ University of Colorado, Boulder (USA) \\ Mizuko Ito \\ University of California, Irvine (USA)
}

\begin{abstract}
RESUMEN: Por aprendizaje conectado se entiende aquella actividad, práctica o experiencia que vincula los intereses de los aprendices con oportunidades académicas, profesionales y cívicas, a través del apoyo y promoción ofrecida por otros y otras. El propósito fundamental de este artículo consiste en situar dicha aproximación, a la vez teórica y aplicada. Para ello, se describe su origen, los principios o ideas fuerza que subyacen a dicha teoría, así como las condiciones sociales y materiales necesarias para implementar prácticas educativas conectadas desde la perspectiva de la equidad relacional. Se describen algunos ejemplos de aprendizaje conectado para, finalmente, señalar lo que consideramos son algunas de las limitaciones de dicho enfoque, así como retos para su desarrollo e implementación.
\end{abstract}

PALABRAS CLAVE: aprendizaje conectado, interés, equidad relacional, cultura participativa.

\section{SOME CHALLENGES, PRINCIPLES AND APPLICATIONS OF THE CONNECTED LEARNING APPROACH}

\footnotetext{
ABSTRACT: Connected learning refers to an activity, practice, or experience that brings together youth interests and learning with academic, career, and/or civic related opportunities - and is supported by and through peer interaction. The fundamental purpose of this article is to situate the approach of connected learning, both in theory and practice. To do so, we describe its origins and
} 
underlying theoretical principles, as well as the requisite social and material conditions to instantiate a connected learning approach in educational settings in ways that enable the promotion of relational equity. In closing, we offer some examples of connected learning in practice, as well as some of what we consider to be its current limitations and design and implementation challenges.

KEYWORDS: Connected learning, interest, relational equity, participatory culture.

Recibido: 02/07/2019

Aceptado: 12/09/2019

Correspondencia: Moisés Esteban-Guitart, Universidad de Girona, Institut De Recerca Educativa, Plaça Sant Domènec, 9, 17004 Girona. E-mail: moises.esteban@udg.edu.

\section{¿EN QUÉ CONSISTE la APROXIMACIÓN DEl “APRENDIZAJE CONECTADO"?}

La aproximación del "aprendizaje conectado" surge en el marco de la iniciativa "Aprendizaje y medios digitales" promovida por la Fundación MacArthur en los Estados Unidos de América (Esteban-Guitart, González-Patiño, y Gee, 2019; Gee, 2010). Inicialmente, la motivación principal fue analizar cómo los medios digitales están transformando los procesos de aprendizaje en las sociedades hiperconectadas del siglo XXI. En este sentido, en el año 2005 se financian distintas investigaciones que podríamos vincular a dos grandes cuestiones. En primer lugar, con el objetivo de entender cómo las personas están cambiando como consecuencia de la utilización de los medios digitales, se promueven estudios etnográficos sobre la participación de los jóvenes en los medios digitales caracterizados por la importancia de las redes sociales, así como la producción y distribución de contenidos culturales (Ito et al., 2009). En segundo lugar, se pretende dar respuesta a la pregunta sobre cómo están cambiando las instituciones cívicas y sociales, en particular los contextos de aprendizaje y, de hecho, cómo deberían ser en el futuro. Aquí podemos situar el estudio liderado por Jenkins a propósito de las nuevas alfabetizaciones mediáticas (proyecto "New Media Literacies") y su impacto en la educación del siglo XXI (Jenkins, 2009). Así como el proyecto "Red digital juvenil" ("Digital Youth Network") que pretende desarrollar habilidades técnicas, creativas y analíticas entre estudiantes de la ciudad de Chicago con riesgo de exclusión social (Barron, Gómez, Pinkard, y Martin, 2014; Pinkard, 2011).

De estos trabajos iniciales se desprenden varias conclusiones y desarrollos teóricos que subyacen a la aproximación del aprendizaje conectado. En particular, se describe cómo las redes sociales, juegos en línea, páginas web de producción y distribución de videos, o aparatos como iPads y móviles forman parte de la cultura cotidiana de jóvenes fans de Harry Potter, jugadores de videojuegos, o creadores de música hip hop. Conceptos como los de géneros de participación, redes públicas, 
aprendizaje basado en la interacción con iguales y en base al interés, ecología de medios o nuevas alfabetizaciones mediáticas se derivan del estudio de la interacción de los jóvenes con una ecología mediática descrita por Jenkins (2006) como "convergente". De modo que lo que se pone en cuestión, en la nueva ecología mediática, es la transformación de la relación entre contenidos, medios y usuarios (González-Patiño y Esteban-Guitart, 2014). En el contexto digital, a partir de la web 2.0, los usuarios pasan a ser potencialmente parte activa/participativa en la recepción, creación y difusión de contenidos culturales. Lo que el mismo Jenkins (1992) describe bajo la etiqueta de "cultura participativa", o Gee (2004) bajo la de "espacios de afinidad".

En este sentido, Ito et al. (2009) diferenciaron tres tipos de participación en función del grado de implicación con los medios. Desde "pasar el tiempo" en actividades de producción e intercambio con iguales con el objetivo de mantener el contacto ("hanging out"); pasando por la búsqueda y manejo de contenidos en la red a partir de un interés o motivación particular ("messing around"), hasta una intensa utilización de los medios que produce cierta especialización en su uso que se traduce, incluso, en procesos de creación de reglas sociales y tecnológicas, así como en el desarrollo de modelos alternativos de estatus y credibilidad como en el caso de grupos de fans ("geeking out"). Por ejemplo, "YOUmedia " es un espacio público, de acceso gratuito, de estudio, creación y participación diseñado para adolescentes con el objetivo de que puedan implicarse en estos tres géneros de participación. Desde pasar el tiempo con iguales, buscar y combinar contenidos en libros y en la red, hasta llevar a cabo proyectos de creación de música propia, videos, diseño $2 \mathrm{D}$ y $3 \mathrm{D}$, videojuegos o fotografía con la ayuda de mentores más especializados (VV.AA., 2014).

Esta participación no se suscribe exclusivamente al ámbito de la creación colectiva de productos o artefactos culturales, lo que podríamos Ilamar "producción colaborativa" (Ito et al., 2019), sino también formas de práctica compartida vinculadas a la competición y la organización comunitaria. Por ejemplo, en el caso de comunidades de danza Bollywood, a través de las redes, y encuentros presenciales, se producen coreografías y competiciones, a nivel regional y nacional, en los Estados Unidos de América, además de llevarse a cabo procesos de organización de la comunidad a través de paginas de Facebook como "desidanceteams", y otras plataformas especializadas como desidancenetwork.org. "Prepararse para una competición es un esfuerzo importante que incluye no solamente producir coreografías y practicar las danzas, sino también recaudar fondos, crear vídeos y accesorios de vestimenta varia" (Ito et al., 2019, p. 59).

Una de las ideas que subyace en estos trabajos es, precisamente, la ilustración de procesos de aprendizaje "espontáneo" que se activan y mantienen a partir de un interés, del apoyo de otros y otras, así como la utilización de habilidades transmedia que permiten construir una historia mediante la vinculación de distintos medios y prácticas socioculturales diversas (Jenkins, 2009).

En este contexto, en el año 2013 se publica el informe Connected learning: An agenda for research and design, donde se define el aprendizaje conectado como la 
vinculación de un interés personal con un contenido o competencia académica, cívica o desarrollo profesional, mediante la asistencia y ayuda de otros y otras. "El aprendizaje conectado se realiza cuando un joven es capaz de extender y vincular, con el apoyo de sus amigos o adultos, una pasión o interés personal con el aprendizaje académico, implicación cívica o carrera profesional" (Ito, et al., 2013, p. 4). De manera que a través de condiciones sociales de apoyo y colaboración se espera conectar los intereses de los aprendices con las oportunidades de aprendizaje disponibles, ya sean éstas online $u$ offline.

Por ejemplo, en la ciudad de Chicago surge la iniciativa "Cities of Learning" en la que niños/as, adolescentes y jóvenes pueden elegir entre una amplia variedad de oportunidades de aprendizaje recogidas en una web que facilita la participación en actividades reconocidas mediante "insignias digitales" basadas en su nivel de participación, como "Investigador científico" ("Science Research"), "Mentor" ("Peer Mentor") o "Instructor de robots" ("Robot instructions"). Tras crear un perfil de usuario, el entorno web permite explorar distintas actividades online y/o offline recogidas en once áreas de aprendizaje: Juegos/codificación, acción comunitaria, diseño, ciencias de la tierra, medios, números, actuación, deporte, narración de historias, trabajo/carrera profesional y zoología. Bajo la entrada libre "Estoy interesado en:" se pueden filtrar las actividades por vecindario, edad, por si son presenciales o online, o si son gratuitas o de pago.

En definitiva, en esta aproximación, a la vez teórica y aplicada, se suponen dos niveles de conexión (Penuel y DiGiacomo, 2017). En primer lugar, aquella que se da, a través de la mediación social (con el apoyo y colaboración de otros y otras), entre los intereses personales y los ámbitos académico, profesional o cívico. En segundo lugar, para que ello pueda darse, se requiere de vínculos entre distintas experiencias, prácticas y contextos de vida y actividad. En este sentido, los medios digitales permiten materializar dichas conexiones y sostener, potencialmente, y bajo ciertas condiciones que expondremos posteriormente, procesos de aprendizaje conectado.

\section{Principios del “APrendizaje ConeCtado"}

A partir del análisis de experiencias educativas como "A Quest to Learn", en la ciudad de Nueva York, la alianza sobre Harry Potter, o el programa YOUMedia, mencionado anteriormente, se propusieron los siguientes principios (Ito et al., 2013): Apoyo entre iguales ("peer-supported"); Aprendizaje estimulado por un determinado interés ("interest-powered") y Orientación académica ("academically oriented"). En este sentido, se argumentaba que experiencias de aprendizaje conectado suponen la utilización de instrumentos digitales para crear y compartir contenidos culturales ("production-centered"), en base a objetivos compartidos ("shared purpose") en plataformas y herramientas digitales que ofrecen recursos de aprendizaje a lo largo y ancho de la vida ("openly networked") (Ito et al., 2013).

A pesar de que estos principios siguen siendo vigentes, recientemente el modelo se encuentra en proceso de reformulación, lo que se ha traducido en la propuesta de los siguientes cuatro principios que pasamos a describir a continuación (ver Figura 1). 


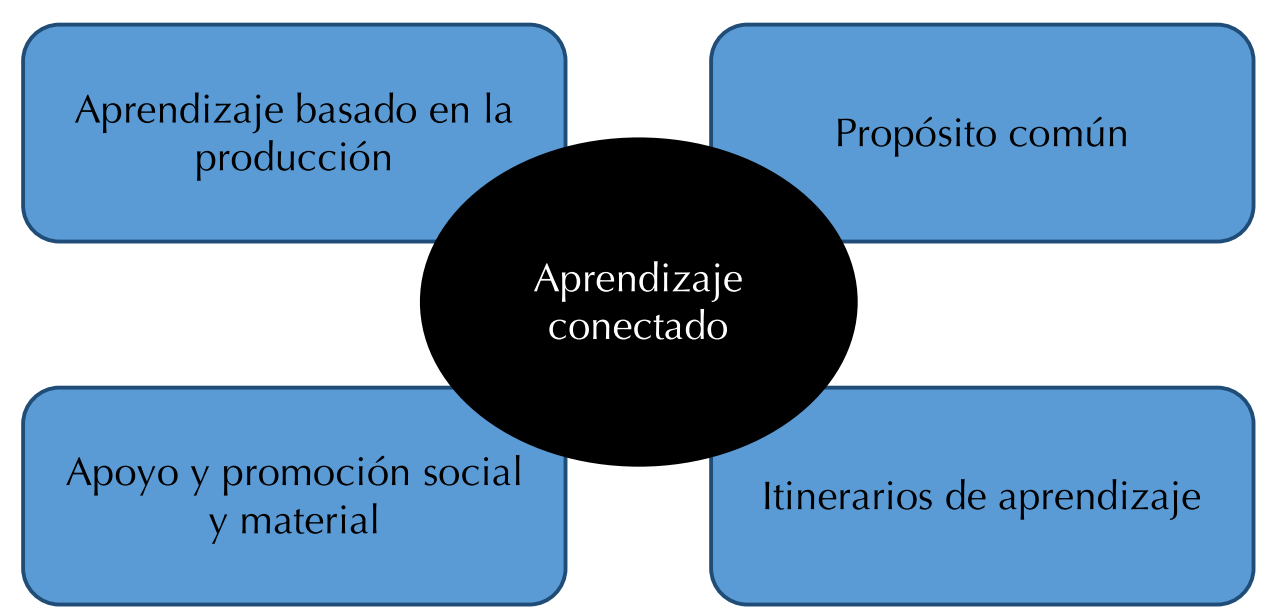

Figura 1. Principios del aprendizaje conectado

\section{Procesos de aprendizaje Centrados en la producción}

Los medios digitales ofrecen una gran variedad de opciones para crear, mezclar y compartir contenidos culturales, descubrir nuevos intereses, así como realizar aprendizajes a partir de la exploración de intereses ya existentes. Sin embargo, el mero acceso a la red no significa automáticamente que el aprendizaje se propicie (Penuel, 2006). Es necesario, en este sentido, disponer de ayuda y orientación para poder utilizar críticamente los medios digitales (Chávez y Soep, 2005), así como para poder utilizarlos en beneficio de procesos de aprendizaje conectado (Ching, Santo, Hoadley y Peppler, 2015; Ito et al., 2019).

Las actividades que se generan en la red, y de hecho en otros espacios físicos de socialización, son a menudo contextos para la autoexpresión y el desarrollo de la identidad (Ito et al., 2019). De ahí que la producción, en sí misma, sea una forma de aprendizaje orientada a la exploración de intereses y su vinculación con oportunidades académicas, cívicas o profesionales. Además, la ubicuidad de los medios digitales disponibles facilita, potencialmente, el acceso a los mismos, así como la difusión de las expresiones culturales creadas.

Una de las formas de visibilizar este principio es el trabajo alrededor de proyectos colectivos que respondan a problemas reales frente a un público también "auténtico" (real). Ello propicia lo que Jurow, Teeters, Shea y Van Steenis (2016) Ilaman "aprendizaje consecuencial" entendido como una acción significativa que se extiende a través del tiempo y el espacio. Es necesario, para ello, que los participantes dispongan de las herramientas y conocimientos que les permitan contribuir significativamente, así como de las condiciones de ayuda social necesarias (DiGiacomo, Van Steenis, Van Horne y Penuel, 2018).

En las ecologías de aprendizaje conectado, el trabajo de los jóvenes participantes a menudo se expresa a través de distintas modalidades y productos como, por ejem- 
plo, historias digitales, diseño gráfico, o llaveros realizados con impresoras 3D. El carácter colectivo de dichos artefactos, así como el hecho que se puedan compartir, es fundamental para que el aprendizaje devenga conectado. Las actividades de producción, en sí mismas, requieren de distintas formas de experiencia, colaboración y retroalimentación. Dicho con otras palabras, la colaboración en la resolución compartida de problemas es una necesidad, más que una restricción, de la actividad, así como su resultado o producto. En el caso de la publicación de una historia digital, por ejemplo, conocimientos y destrezas en entrevistas, así como formas de publicación mediática, permiten la producción exitosa de una historia basada en un interés particular.

A modo de ejemplo, el proyecto "FUSE Studio", implementado en entornos escolares de los Estados Unidos de América, Finlandia e Israel, permite ilustrar dicho principio. Consiste en una amplia variedad de actividades vinculadas a ciencias, tecnología, ingeniería, arte y matemáticas (STEAM). A través de distintos desafíos (por ejemplo "Diseñador de joyas", "Carrera de obstáculos con robots" o "Estructuras espagueti"), online y offline, los estudiantes exploran distintos problemas para subir de nivel. Los retos van acompañados de distintos artefactos como ordenadores, impresoras 3D, goma espuma, cinta, tijeras, así como instrucciones para llevar a cabo el desafío. Dicho entorno de actividades está diseñado para implicar a los estudiantes en distintos temas del área de STEAM. Los estudiantes pueden elegir, a partir de sus intereses, qué desafío quieren afrontar, cuando y con quién. Pueden elegir, por ejemplo, trabajar autónomamente o en grupo. No existe, en dichas actividades, ningún proceso de evaluación por parte de los docentes. A través de la utilización de fotografías, vídeos, así como otros artefactos digitales, son los propios estudiantes que documentan el proceso de desarrollo de un desafío, que permite poder acceder a otro desafío (Kumpulainen, Kajamaa y Rajala, 2018).

Más específicamente, en este particular contexto y actividad, los participantes aprovechan la experiencia de sus compañeros y compañeras para crear, por ejemplo, un nuevo tono de llamada para sus teléfonos móviles. Más importante aún, disponer de esta experiencia colectiva, esta especie de inteligencia colectiva, situada y distribuida, se hace necesaria para poder crear el producto. En este sentido la actividad centrada en la producción es auténtica. En pocas palabras, la colaboración (solicitar ayuda a compañeros y compañeras) es natural y necesaria para completar con éxito la producción del artefacto. Lo que en muchas ocasiones no sucede con las tareas escolares. En las actividades del proyecto FUSE, al igual que en otros contextos de aprendizaje conectado, crear un producto colectivamente para compartirlo en un contexto social de juego, por ejemplo crear un vehículo solar, es un principio rector en el diseño de la actividad. De manera que en dichas actividades, además de trabajar competencias vinculadas a las Ilamadas STEAM, se facilitan la creación/vinculación equitativa de experiencias diversas dentro y fuera del contexto escolar (DiGiacomo, Van Horne y Penuel, 2020). 


\section{Propósito común}

Existe un considerable consenso alrededor de la importancia que tiene el reconocimiento de la voz de los participantes en una actividad sociocultural determinada. De manera que la implicación de los aprendices en los procesos de decisión sobre los objetivos de las actividades en las que están invitados a participar, no solamente permite el desarrollo de la pertenencia y compromiso con la actividad misma, sino también facilita la emergencia de procesos de exploración de nuevas identidades, desarrollo de habilidades sociales y confianza, así como optimiza el aprendizaje, en general (Penuel, Clark y Bevan, 2016). En este sentido, el principio del propósito común parte de la necesidad de reconocer la construcción compartida de objetivos, intereses y prácticas en cualquier diseño de aprendizaje conectado. Objetivos compartidos que, en algunos casos, pueden ser tan ambiciosos como la mejora y transformación social. Por ejemplo, la comunidad "Nerdfighter" surge de un canal de YouTube creado por los hermanos John y Hank Green con el propósito de fomentar procesos positivos de cambio social, promocionando actuaciones solidarias y de activismo social. Lo que permite ilustrar procesos de "política participativa" caracterizada por actuaciones interactivas entre grupos de iguales que persiguen tener voz, participación e influencia en temas de interés social, público y comunitario (Ito et al., 2019; Jenkins, Shresthova, Gamber-Thompson, KliglerVilenchik y Zimmeraman, 2016).

De hecho, como ya se ha dicho anteriormente, los medios y dispositivos digitales hoy disponibles ofrecen una gran variedad de oportunidades de aprendizaje en espacios y actividades a partir de las cuales uno puede participar a partir de una afinidad -interés- común (Gee, 2005; Gee y Esteban-Guitart, 2019; Ito et al., 2019). Estos escenarios/actividades, a pesar de que no suelen restringir el acceso por edad, género u otra condición, como sucede en la escuela, suelen tener normas compartidas que pueden generar conflictos no solamente en el acceso y la participación, sino también a nivel de valores y normas aceptadas en dicho espacio de afinidad (Garcia, 2017).

En este sentido, los espacios y actividades que persiguen fomentar procesos equitativos de aprendizaje conectado necesitan, por una parte, ser acogedores. Es decir, facilitar el acceso y contribución de los nuevos participantes. Además, deben poder facilitar la contribución de dichos participantes en el mantenimiento y cambio de las normas y códigos del espacio de afinidad. Eso se traduce en poder adoptar distintos roles, así como no penalizar los distintos niveles de experiencia como sucede en la escuela y los contextos formales de enseñanza y aprendizaje. Por otra parte, dichos entornos deben sostener las prácticas culturales, de sentido y valor de los participantes (Esteban-Guitart, Lalueza, Zhang-Yu y Llopart, 2019; Paris, 2012). Es decir, deben disponer de mecanismos para poder incorporar los fondos de conocimiento e identidad de los aprendices (Esteban-Guitart, 2016), es decir, sus destrezas, saberes previos, así como intereses y referentes de sentido e identificación. Con ello, se facilita la creación de nuevos contextos y prácticas híbridas que dinámicamente se construyen a partir de la actividad de los participantes (Esteban-Guitart, 2010; Gutiérrez y Rogoff, 2003; Rogoff, 2016). 


\section{El APOYO Y PROMOCIÓN}

Brand (1998) propuso la noción de "sponsorship" (que aquí traducimos como "apoyo y promoción") para describir cómo se organiza el acceso a prácticas de alfabetización en el contexto escolar. En este sentido, los "sponsors": "establecen las condiciones de acceso a la alfabetización, así como ofrecen incentivos para mantener el compromiso. La historia muestra que los procesos de alfabetización han estado siempre acompañados de procesos de permiso, sanción, asistencia, coerción" (Brandt, 1998, pp. 166-167).

Cabe decir aquí que los contextos y actividades de aprendizaje no están necesariamente conectados entre sí. Conectar el participante a nuevas oportunidades de aprendizaje (contextos, actividades, recursos) requiere de cierta ayuda y asistencia para superar las barreras de acceso a las mismas. La idea de "patrocinio" ("sponsorship") supone reconocer el papel activo de otros y otras en la posibilidad de crear vínculos entre aprendices y oportunidades de aprendizaje. Puede conllevar, por ejemplo, conectar un joven con una oportunidad de desarrollo profesional (trabajo), facilitar el reconocimiento social de sus competencias, ayudarle a construir una red social de apoyo, o facilitarle el acceso a recursos e instrumentos necesarios para desarrollar sus intereses (Allen, DiGiacomo, Van Horne y Penuel, 2018; Barron et al., 2010). Puede conllevar, también, apoyo social y económico, especialmente necesario para superar las condiciones estructurales de inequidad (Garcia y O'Donnel-Allen, 2015). El apoyo emocional es también un elemento imprescindible en los procesos de apoyo y patrocinio en el marco de experiencias basadas en el aprendizaje conectado.

Existen ciertas condiciones que pueden fomentar los procesos de apoyo y promoción en contextos y situaciones de aprendizaje conectado. En primer lugar, en el contexto de un espacio online de afinidad, mentores e iguales pueden visibilizar las rutas necesarias para desarrollar cierta pericia. Pueden también establecer normas que permitan facilitar el acceso a los nuevos participantes, así como sentirse aceptados y bienvenidos. En segundo lugar, jóvenes y adultos que disponen de redes sociales ricas y amplias, así como recursos y saberes, son buenos candidatos en la ayuda y promoción de proyectos de aprendizaje de otras personas. Una estrategia efectiva se vincula al diseño para el patrocinio ("design for brokering"), es decir, crear oportunidades para que determinadas personas puedan actuar como traductoras al facilitar puentes (transiciones) entre dominios y contextos. Otro elemento clave para el apoyo y promoción es facilitar el espacio y recurso necesario para poder desarroIlar un determinado interés (Barron, 2010). La falta de herramientas y recursos, o la imposibilidad de acceder a ellos, suele interrumpir la realización de actividades de aprendizaje basadas en un determinado interés (DiGiacomo, Van Steenis, Van Horne y Penuel, 2018). Otro aspecto vinculado con los procesos de apoyo y promoción consiste en la conexión del aprendiz con los recursos, servicios y actividades en curso de la comunidad a través de mostrar públicamente su trabajo, o contribuir a procesos de cambio y transformación social (Pennuel y O'Connor, 2018). Ello puede conferir dignidad a los propósitos de aprendizaje, legitimando sus intereses, valores y prácticas (Espinoza y Vossoughi, 2014). 


\section{LA CONSTRUCCIÓN DE ITINERARIOS DE APRENDIZAJE}

Desde la perspectiva que adoptamos, el aprendizaje es un proceso que se desarrolla a través de la participación en distintas actividades socioculturales, en distintos momentos temporales (Esteban-Guitart, Coll y Penuel, 2018). Lo que nos lleva a la noción de itinerario, no entendida como la unión de un punto inicial y uno final, sino más bien como el transcurso de situaciones que se desencadenan en dis(continuidades) entre distintas experiencias de aprendizaje. En el marco de la aproximación del aprendizaje conectado, ello supone reconocer que la intervención educativa se encamina a facilitar itinerarios para explorar y desarrollar intereses conectados con oportunidades cívicas, académicas o profesionales.

La palabra "construcción" connota el trabajo activo necesario para ello, tanto por parte de los jóvenes participantes como el grupo de iguales y adultos de apoyo. Por supuesto, construir itinerarios o caminos de aprendizaje supone encontrar oportunidades existentes, pero a menudo implica la necesidad de crear nuevas oportunidades (Barron, 2010). En este sentido, que una comunidad disponga de un abanico heterogéneo y amplio de oportunidades es un indicador de los itinerarios de aprendizaje que potencialmente se puedan construir. Es decir, la existencia de programas, servicios y recursos educativos (cantidad de ellos) es importante. Lo es también, por supuesto, la calidad de los mismos.

Otro rasgo que la literatura ha mostrado ser significativo para la optimización de los procesos de aprendizaje alude a la "redundancia" (Super y Harkness, 2002), es decir, la existencia de múltiples influencias similares -"redundantes"- en un mismo período de desarrollo en distintos contextos de vida y actividad. Estos caminos, trayectorias o itinerarios de aprendizaje necesitan sistemas de reconocimiento de los desarrollos de los participantes para visibilizar sus logros.

Recientemente, por ejemplo en el contexto del "Chicago City of Learning", se han propuesto credenciales digitales para reconocer los conocimientos y habilidades que jóvenes han desarrollado en distintos contextos. La idea subyacente es que una insignia, o credencial digital, puede abrir la posibilidad de participar en contextos y prácticas de aprendizaje de mayor dificultad. En los sistemas abiertos de credenciales o insignias de aprendizaje, los participantes pueden visibilizar rutas, caminos o trayectorias de aprendizaje, obteniendo orientación en relación a la credencial que necesitarían adquirir para progresar (O'Byrne, Schenke, Willis y Hickey, 2015). Por ejemplo, el taller "Diseño de un salón tecnológico para tu escuela", ofrecido por la Fundación de Arquitectura de Chicago, permite transformar un laboratorio viejo a un espacio de aprendizaje digital. Es una actividad gratuita, online, para niños y niñas de 13 a 18 años de edad, que permite obtener distintos diplomas ("digital badges") como "Dibujo de construcción", niveles 1, 2 y 3, o "Fabricación de modelos digitales", entre otros.

Finalmente, al igual que sucede con los otros principios, los aprendices necesitan acceder a las herramientas y recursos para poder proseguir con sus intereses y vincularlos a sus motivos académicos, profesionales y/o cívicos. Particularmente relevante nos parece la posibilidad de compartir portafolios digitales de trabajo con otros, así 
como posibilidades de re-acceso y re-conexión con las actividades de aprendizaje basadas en los intereses de los aprendices cuando éstas se interrumpen.

No es fácil diseñar situaciones y experiencias que puedan satisfacer dichos principios. Sin embargo, existen ejemplos empíricos de contextos y actividades que han permitido ilustrar su implementación, y que hemos descrito anteriormente ("YOUMedia", "Cities of Learning", "FUSEstudio"). Dichos ejemplos comparten la atención programática a las condiciones sociales y materiales necesarias para que el aprendizaje conectado pueda darse a distintos niveles: actividad, interacción y contexto. En otras palabras, lo que hace que cada uno de estos ejemplos empíricos pueda considerarse un ejemplo de aprendizaje conectado es que los jóvenes pueden participar, en los espacios determinados, en actividades, relaciones y contextos socioculturales extendidos para: 1) poder explorar sus intereses con compañeros/as y mentores o tutores con más experiencia que ellos/ellas, 2) colaborar y recibir comentarios y feedback para profundizar, ampliar o cambiar su compromiso dentro de un dominio particular alienado con un determinado interés, 3) sentirse parte de una práctica, mediada digitalmente, que permite la apropiación de determinados artefactos como, por ejemplo, la creación de una canción, una maqueta o un blog particular. En definitiva, se requieren de condiciones sociales y materiales para poder materializar procesos de aprendizaje conectado. Lo que a continuación pasamos a describir con una mayor profundidad.

\section{Condiciones PARA el APRENDIZAJE CONECTADO DESDE LA EQUidAD RELACIONAL}

Uno de los retos más importantes que tienen ante sí los sistemas y políticas educativas es, a nuestro entender, garantizar el pleno acceso a las oportunidades, espacios y sistemas distribuidos de aprendizaje. Especialmente nos parece crítico dicho acceso para las personas y grupos socioculturales infrarepresentados en la cultura escolar de la sociedad dominante en un determinado territorio. De modo que el diseño de experiencias y prácticas debe atender a la distribución del acceso a los recursos físicos y digitales, así como las oportunidades que potencialmente proviene un determinado contexto, práctica o actividad.

Específicamente, se ha sugerido la noción de "equidad relacional" para hacer referencia a aquellas actividades o experiencias que permiten a los participantes contribuir conjunta y colectivamente, en condiciones de igualdad, en los objetivos y tareas de aprendizaje (DiGiacomo y Gutiérrez, 2015). Además de poder reflexionar, críticamente, sobre la comprensión de uno mismo, el mundo y la relación entre uno mismo y lo que le rodea (Penuel y DiGiacomo, 2018).

Para facilitar procesos de aprendizaje conectado basados en la equidad relacional, se requieren de ciertas condiciones sociales y materiales. Se trata de propiciar el diseño de espacios que conjuntamente puedan constituir ecologías de aprendizaje socioespacial más amplias a través de las cuales puedan materializarse los principios anteriormente descritos. Si bien no es una lista exhaustiva, pensamos que las siguientes condiciones son especialmente significativas para el diseño y organización de 
procesos de aprendizaje conectado (Penuel, Chang-Order y Michalchik, 2018). Se trata de condiciones que, de una forma más explícita o menos, hemos mencionado anteriormente y que pasamos a describir, brevemente, a continuación.

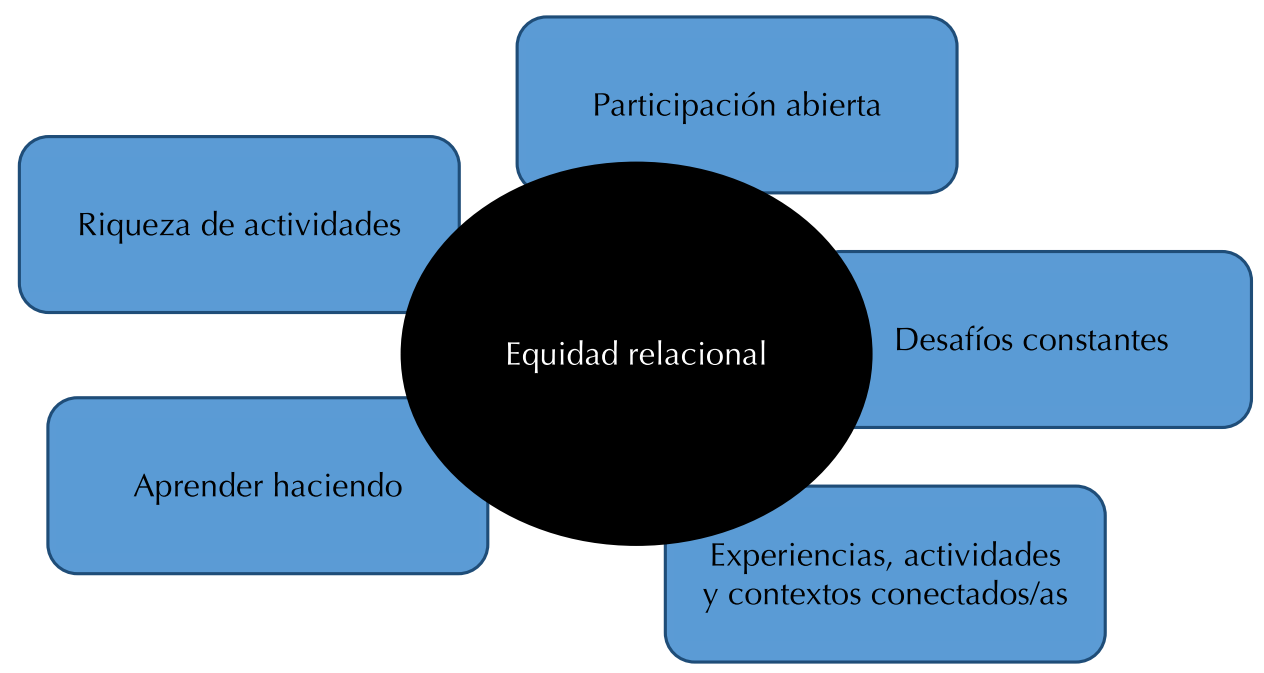

Figura 2. Condiciones para el aprendizaje conectado desde la equidad relacional

Riqueza de actividades. Es necesario que en los espacios/sitios, ya sean virtuales o físicos o una combinación de ambos, se incluya un amplio repertorio de actividades vinculadas a los intereses de los aprendices que permitan su elección, participación e implicación. Dichas actividades deben ser intencionalmente diversas en cuanto a dominio y medio.

Participación abierta. Las actividades deben ofrecer múltiples vías de acceso, así como también invitar a la contribución de distintas personas con distintos niveles de experiencia y conocimiento. De modo que se debe permitir el desarrollo de distintas maneras de experimentar el sentido de pertenencia social: desde asumir un rol de experto, hasta crear y compartir comentarios, experiencias o productos, o convertirse en autor para mostrar un trabajo, por ejemplo una canción, en un contexto de audiencia real. Además deben contemplarse distintas modalidades de implicación como, por ejemplo, el trabajo independiente, colaborativo, o ambos, que faciliten la exploración y desarrollo de intereses.

Aprender haciendo. Para que el contexto o actividad esté basado en la producción se requiere que los participantes se impliquen en alguna forma de enseñanzaaprendizaje basada en la experiencia. Dicho con otras palabras, el aprendizaje no debe reducirse a la adquisición de conocimientos, o a la mera participación en actividades con sentido, sino también a la producción de experiencias. En entornos que promueven el aprendizaje conectado, los jóvenes se implican, movidos por un interés particular, en el diseño de actividades, la escritura y representación de textos, el registro de vídeos o la codificación y creación de juegos. 
Desafíos constantes. El entorno de enseñanza y aprendizaje debe fomentar retos asumibles pero que lleven a los aprendices más allá de lo que pueden hacer por sí mismos autónomamente. Lo que facilita la creación de "zonas de desarrollo próximo" (Vygotsky, 1963) en las que se hace necesaria la participación de artefactos o personas para andamiar el proceso de resolución de la tarea o actividad.

Experiencias, actividades y contextos conectados/as. Se asume la premisa según la cual el aprendizaje es un fenómeno que se desarrolla a través del tiempo y del espacio (Esteban-Guitart, Coll, y Penuel, 2018). Dicho con otras palabras, cuando una persona se implica en la consecución de un interés, se implica y participa en distintas actividades y contextos a lo largo del tiempo. De modo que se debe facilitar, a la vez, la conexión de experiencias de aprendizaje, y contextos/prácticas facilitando su acceso y participación.

\section{AlCance y limitaciones}

Según la base de datos ERIC (consultada el 5/10/2018) existen 800 publicaciones revisadas por pares en los últimos cinco años (2014-2018) bajo la etiqueta "connected learning". Algunos de los principales descriptores de estos trabajos, según dicha base de datos, son: métodos de enseñanza, investigación cualitativa, aprendizaje de segundas lenguas, estudios de caso o educación tecnológica, lo que permite hacerse una idea general sobre los principales contenidos que se abordan en dichos trabajos. En cuanto al nivel educativo, destaca los trabajos vinculados a la educación superior (314 en total), seguidos de trabajos llevados a cabo en educación postsecundaria (225), educación secundaria (112) y educación primaria (102), lo que muestra que la aproximación se ha aplicado básicamente con adolescentes y jóvenes. Finalmente, a nivel general descriptivo, destaca el carácter internacional de dichos trabajos, pues se han Ilevado a cabo, fundamentalmente, en Estados Unidos de América, pero también en Australia, Canadá, Europa (Alemania, Finlandia, Suecia, Reino Unido, Holanda, Noruega y España), y en menor medida en: China, Turquía, Japón, Sudáfrica, Rusia, Nueva Zelanda, Singapur, Indonesia o Israel.

No pretendemos, en este artículo, llevar a cabo una revisión exhaustiva y profunda de dicha literatura, pero sí que podemos sacar algunas conclusiones a partir de ella, así como identificar algunas líneas de trabajo que nos parecen especialmente relevantes en el marco de la aproximación del aprendizaje conectado. En particular vamos a destacar una línea de investigación vinculada al análisis sobre la intersección entre intereses y oportunidades, así como los aspectos sociales vinculados a dicha conexión.

La aproximación del aprendizaje conectado puede utilizarse como marco heurístico para investigar el diseño de actividades de aprendizaje cuando se vinculan los intereses de los aprendices con oportunidades futuras de desarrollo académico, cívico, profesional. El establecimiento de redes sociales de conexión y apoyo y promoción parecen ser claves aquí. Martin (2018), por ejemplo, utilizó el marco del aprendizaje conectado para explorar "la intersección entre interés y oportunidad" 
(p. 131) en jóvenes usuarios de Scratch¹. En particular, el estudio de Martin muestra que no importa tanto el punto de acceso a la actividad, ni el contexto sobre el cual se desarrolla inicialmente la misma (la escuela, extraescolar, familia, amigos), sino sobre todo: 1) el apoyo por parte de iguales, 2) la cura por parte de adultos y 3 ) el acceso a entornos sociales que no solamente estimulan los intereses sino que invitan a su exploración. Estos factores se postulan como cruciales para el desarrollo de habilidades de codificación, así como para proyectar carreras profesionales potencialmente vinculadas a las ciencias computacionales. De manera que tras examinar el diseño de una actividad de aprendizaje determinada, Martin concluye enfatizando la centralidad de las relaciones sociales como bisagra para integrar y articular los intereses con las oportunidades.

En esta línea cabe destacar el trabajo de Allen, DiGiacomo, Van Horne y Penuel (2018) que persigue comprender mejor la textura del apoyo y promoción social en experiencias de aprendizaje conectado. Los autores, en este caso, estudiaron cómo jóvenes describen el acceso a actividades vinculadas a su particular itinerario académico. Se trata de un estudio longitudinal sobre actividades basadas en el interés que pone en consideración las circunstancias sociales inmediatas, más que los intereses individuales, como el factor mediador principal en la implicación de los jóvenes en experiencias de aprendizaje conectado. Según concluye el estudio: "Inicialmente destaca una necesidad, por ejemplo vinculada al ámbito profesional, como desencadenante a la participación en una determinada actividad. Sin embargo, los participantes se involucran en la misma gracias el apoyo y patrocinio de otra persona como un docente, padre o amigo" (p. 124). Esta conclusión es consistente con otros trabajos que analizan los procesos de aprendizaje en el siglo XXI (Bender y Peppler, 2019; DiGiacomo, Van Horne, Van Steenis y Penuel, 2018; Erstad, Gilje y Arnseth, 2013) y reorientan el análisis hacia las realidades sociales y materiales que dan forma a las actividades relacionadas con los intereses de los jóvenes al convertirse en claves para entender cómo y por qué los jóvenes buscan experiencias de aprendizaje conectado.

En relación a las limitaciones nos gustaría destacar dos aspectos que nos parecen potencialmente problemáticos. El primero alude a la propia noción de interés y el riesgo de sobredimensionar dicho constructo. El segundo se refiere a la centralidad en la población adolescente y juvenil como diana de la intervención basada en la aproximación del aprendizaje conectado.

Existe un amplio consenso en la literatura disponible alrededor de la vinculación entre el interés y el aprendizaje, actuando éste como un factor psicológicomotivacional que garantiza la implicación con un objeto, idea o actividad a lo largo del tiempo (Barron, 2006; Harackiewicz, Smith y Prinski, 2016; Hidi y Renninger, 2006). Aspecto que se asume en la aproximación del aprendizaje conectado (Ito et al., 2009). Sin embargo, pensamos que existe el riesgo de sobredimensionar el interés reduciéndolo al inicio y final del proceso educativo. Dicho con otras palabras, considerar el interés como educativo en sí mismo (Renninger y Hidi, 2016). Contrariamen-

1. Scratch es un lenguaje de programación visual gratuito y una comunidad en línea donde los usuarios pueden crear historias, juegos y animaciones desarrollado por el grupo Lifelong Kindergarten del MIT Media Lab (Cambridge, Massachusetts, USA). Ver: https://scratch.mit.edu/explore/projects/games/ 
te, pensamos que el interés no debería equivaler exclusivamente a una preferencia, algo placentero, una opción personal, o un resultado conseguido, sino algo transitorio, incierto, un mero punto inicial hacia una experiencia de aprendizaje más rica y variada (Dewey, 1913). Según Vygotsky (1997): "Es necesario que el contexto sea un asunto personal del alumno, y solamente entonces podremos estar seguros de tener éxito. De un interés del niño a otro nuevo interés, esta debería ser la regla" (p. 86). En este sentido, la mediación social o la actividad, experiencia misma, debería diseñarse con el objetivo de partir de las necesidades, identidades e intereses del aprendiz para facilitar la construcción de nuevas necesidades, identidades e intereses. En este sentido, podríamos definir el aprendizaje como un proceso de transformación identitaria (reconocerse, social y personalmente, como actor, escritor, científico, músico, matemático o profesor, más que hacer matemáticas, literatura o arte) movilizado por la co-construcción de necesidades e intereses. Intereses, por otra parte, que pensamos deberían conducirse hacia objetivos colectivos deseables como la sostenibilidad, equidad, cohesión y justicia social, más que aspiraciones puramente personales.

La otra crítica que nos gustaría destacar aquí es que, básicamente, se han diseñado contextos o situaciones de aprendizaje conectado pensando en jóvenes y adolescentes. Desde una perspectiva inclusiva y holística del aprendizaje, sin embargo, éste se da a lo largo de la vida, de modo que se requiere de intervenciones más amplias que contemplen mayores rangos de edad.

\section{Algunos retos para considerar. A modo de conclusión}

Como se desprende de las secciones precedentes, el aprendizaje conectado es un fenómeno multidimensional, multiespacial que se desarrolla a través del tiempo. A pesar de que la experiencia de aprendizaje conectado puede promoverse, inicialmente, en un solo espacio o actividad que permite vincular intereses, personas y oportunidades, el impacto y desarrollo de la misma se sostiene en la medida que organiza la vida de los aprendices hacia cuotas de mayor aprendizaje y mejor bienestar, personal y colectivo, presente y futuro (Gee y Esteban-Guitart, 2019). Dicho con otras palabras, en esta perspectiva las dimensiones entre aprendizaje y vida se diluyen, de modo que el aprendizaje se concibe como un proyecto de vida implicado en procesos de transformación identitaria: devenir(ser) artista, profesor, periodista, científico o escritor de novelas de ficción.

Ello nos lleva a sugerir la necesidad que cualquier investigación sobre experiencias y resultados de procesos de aprendizaje conectado deba ir acompañado de un análisis profundo de las condiciones biográficas, sociales, culturales e incluso políticas que median la naturaleza del aprendizaje (Esteban-Guitart, 2016; Penuel y DiGiacomo, 2018). Además de que dicho análisis debe permitir rastrear el camino, trayectoria y procesos de (dis)continuidad que caracterizan los procesos de aprendizaje (Bronkhorst y Akkerman, 2016). Para ello, se requieren de instrumentos y estrategias metodológicas sensibles a las distintas manifestaciones y dimensiones del aprendizaje conectado, así como su carácter situado y distribuido a lo largo y ancho de la vida. Por ejemplo, el análisis retrospectivo de procesos de aprendizaje (Barron, 2006; Bender y Peppler, 2019) no solamente con entrevistas en profundi- 
dad sino también mediante la creación de "artefactos identitarios" por parte de los propios aprendices (Subero, Llopart, Siqués y Esteban-Guitart, 2018) o "cartografías" que permiten rastrear los espacios, momentos, movimientos y tiempos significativos de aprendizaje (Hernández-Hernández, Sancho-Gil y Domingo-Coscollola, 2018). Se trata de artefactos, con valor epistémico, que permiten, potencialmente, crear conexiones entre contextos y experiencias de aprendizaje (Subero, Vujasinovic y Esteban-Guitart, 2017).

Una perspectiva teórica que nos parece especialmente relevante en el diseño metodológico para el aprendizaje conectado es la teoría de la práctica social (Dreier, 2009). Dicho enfoque sitúa la primera persona en el estudio de la actividad humana como necesaria pero inextricablemente unida a la práctica. Desde esta perspectiva, comprender las acciones que realizan los jóvenes en sus "contextos vitales de aprendizaje" -"learning lives" (Erstad y Sefton-Green, 2013)- requiere de una atención continuada a los procesos de dotación de sentido, así como los intereses y oportunidades que emergen en sus vidas cotidianas (Holzkamp, 2013). Una implicación metodológica de esta teoría, en el campo específico del aprendizaje conectado, es la realización de entrevistas bajo el supuesto de que las personas están situadas a lo largo y ancho de la vida. En este sentido, Penuel, DiGiacomo, Van Horne y Kirshner (2016) han utilizado dicho marco para analizar y comprender la intersección entre intereses y oportunidades en experiencias de aprendizaje conectado en jóvenes.

En realidad esto nos lleva a una conclusión general pero estimamos valiosa. Los procesos de aprendizaje conectado requieren de ecologías situadas y distribuidas de enseñanza-aprendizaje. Espacios-actividades que reconozcan, legitimen y fomenten los intereses e identidades de los aprendices, antes que tacharlos/las de frívolos/as y superficiales. Ello conlleva incorporar y enlazar el contexto-situación-actividad con otros contextos-situaciones-actividades significativas del aprendiz. Lo que diluye las fronteras tradicionales entre el ámbito formal y el ámbito informal (Esteban-Guitart, Coll y Penuel, 2018). Además de crear condiciones para favorecer la colaboración entre iguales, así como procesos de patrocinio (apoyo y promoción de itinerariosproyectos de vida), que vayan más allá de la mentoría, en el sentido que faciliten el acceso a los recursos, servicios y agentes de aprendizaje de una determinada comunidad o territorio. Lo que incluye facilitar las condiciones materiales de acceso, por ejemplo, condiciones económicas.

Finalmente, en la lógica de la ética relacional, brevemente expuesta anteriormente, es necesario que el aprendiz tome voz, participación y decisión en los procesos de enseñanza-aprendizaje en el que está envuelto. Ello significa que los objetivos y prácticas deben basarse en proceso de co-diseño que permitan compartir objetivos de aprendizaje, así como participar en el diseño de las propias actividades de aprendizaje. Significa también que el aprendiz debe adoptar un rol de contribuidor-participante en "esfuerzos auténticos", es decir, en situaciones donde pueda tener algo que decir, así como permitan prepararlo para la acción en otros contextos o situaciones (Penuel, Clark y Bevan, 2016).

De nuevo, para que lo dicho anteriormente suceda, se requiere de personas, con un rol de "conectoras", que faciliten la transición de un contexto, situación, activi- 
dad a otro/a, expandiendo conocimiento procedimental vinculado con trámites burocráticos, acceso a recursos y servicios o requerimientos educativos implicados en determinadas oportunidades de desarrollo académico, cívico, profesional. Pero las "personas conectoras" deben también facilitar "conocimiento espacial" ("know whe$\mathrm{re}^{\prime \prime}$ ): saber las redes de personas, servicios y lugares que el aprendiz debe "visitar" y transitar para desarrollar ciertos aprendizajes.

Sin embargo, a pesar de que el corpus empírico alrededor del aprendizaje conectado va en aumento, no conocemos aun suficientemente la estructura, forma, agentes y condiciones específicas que deben cumplir estas ecologías situadas y distribuidas de enseñanza y aprendizaje para promover itinerarios de aprendizaje profundo, con impacto positivo en la vida personal y colectiva. Tampoco se ha discutido en profundidad el potencial papel de la escuela en una ecología de aprendizaje que trasciende sus fronteras, así como su gramática tradicional. Conocer con más detalle los elementos necesarios o desencadenantes de estos itinerarios de aprendizaje nos debe ayudar al diseño de ecologías de enseñanza y aprendizaje que brinden más y mejores oportunidades educativas a todos los aprendices, más allá de su origen y característica intelectual, económica y social; así como a resituar la escuela en una constelación educativa más amplia reconfigurando su práctica y función principal.

\section{REFERENCIAS BIBLIOGRÁFICAS}

Allen, C., DiGiacomo, D., Van Horne, K. y Penuel, W. R. (2018). Pursuing interests and getting involved: Exploring the conditions of sponsorship in youth learning. Digital Education Review, 33, 120-129. https://doi.org/10.1344/ der.2018.33.120-129.

Barron, B. (2006). Interest and self-sustained learning as catalysts of development: A learning ecology perspective. Human Development, 49, 193-224. https://doi. org/10.1159/000094368.

Barron, B. (2010). Conceptualizing and tracing learning pathways over time and setting. En W. R. Penuel y K. O'Connor (Eds), Learning research as a human science. National Society for the Study of Education Yearbook, 109(1), 113-127.

Barron, B., Gomez, K., Pinkard, N. y Martin, C. (2014). The Digital Youth Network. Cultivating Digital Media Citizenship in Urban Communities. Cambridge, MA: The MIT Press.

Barron, B., Levinson, A., Martin, C. K., Merti, V., Stringer, D., Austin, K., ... Gomez, K. (2010). Supporting young new media producers across learning spaces: A longitudinal study of the Digital Youth Network. En K. Gomez, L. Lyons, y J. Radinsky (Eds.), Learning in the Disciplines: Proceedings of the 9th International Conference of the Learning Sciences (Vol. 2, pp. 203-211). Chicago, IL: International Society of the Learning Sciences.

Bender, S. y Peppler, K. (2019). Connected learning ecologies as a emerging opportunity through Cosplay. Comunicar, 58, 31-40. https://doi.org/10.3916/C582019-03. 
Brandt, D. (1998). Sponsors of literacy. College Composition and Communication, 49(2), 165-185.

Bronkhorst, L. H., y Akkerman, S. F. (2016). At the boundary of school: Continuity and discontinuity in learning across contexts. Educational Research Review, 19, 18-35. https://doi.org/10.1016/j.edurev.2016.04.001

Chávez, V. y Soep, E. (2005). Youth Radio and the pedagogy of collegiality. Harvard Educational Review, 75(4), 409-434. https://doi.org/10.17763/ haer.75.4.827u365446030386.

Ching, D., Santo, R., Hoadley, C. y Peppler, K. A. (2015). On-Ramps, Lane Changes, Detours and Destinations: Building Connected Learning Pathways in Hive NYC through Brokering Future Learning Opportunities. New York, NY: Hive Research Lab.

Dewey, J. (1913). Interest and Effort in Education. Chicago, IL: The Riverside Press Cambridge.

DiGiacomo, D., Van Horne, K. y Penuel, W. R. (2020). Choice and interest in designed learning environments: the case of FUSE Studios. Information and Learning Sciences, 131(3/4), 137-154. https://doi.org/10.1108/ILS-09-2019-0098.

DiGiacomo, D., Van Steenis, E., Van Horne, K. y Penuel, W. R. (2018). The material and social constitution of interest. Language, Culture, and Social Interaction, 19, 51-60. https://doi.org/10.1016/j.Icsi.2018.04.010.

DiGiacomo, D. K. y Gutiérrez, K. D. (2015). Relational equity as a design tool within making and tinkering activities. Mind, Culture, and Activity, 23(2), 141-153. https://doi.org/10.1080/10749039.2015.1058398.

Dreier, O. (2009). Persons in structures of social practice. Theory \& Psychology, 19(2), 193-212. https://doi.org/10.1177/0959354309103539.

Erstad, O., Gilje, Ø. y Arnseth, H. C. (2013). Learning lives connected: Digital youth across school and community spaces. Comunicar, 40, 89-98. https://doi. org/10.3916/C40-2013-02-09.

Erstad, O. y Sefton-Green, J. (Eds.). (2013). Identity, Community, and Learning Lives in the Digital Age. New York, NY: Cambridge University Press.

Espinoza, M. y Vossoughi, S. (2014). Perceiving learning anew: Social interaction, dignity, and educational rights. Harvard Educational Review, 84(3), 285-313. https://doi.org/10.17763/haer.84.3.y4011442g71250q2.

Esteban-Guitart, M. (2010). Geografías del desarrollo humano. Una aproximación a la psicología cultural. Barcelona: Aresta.

Esteban-Guitart, M. (2016). Funds of Identity. Connecting Meaningful Learning Experiences In and Out of School. New York, NY: Cambridge University Press.

Esteban-Guitart, M., Coll, C. y Penuel. W. (2018). Learning across settings and time in the Digital Age. Digital Education Review, 33, 1-25. https://doi.org/10.1344/ der.2018.33.\%25p. 
Esteban-Guitart, M., González-Patiño, J. y Gee, J. (2019). Digital media and learning. Emergent forms of participation and social transformation. Comunicar, 58, 1-5.

Esteban-Guitart, M., Lalueza, J. L., Zhang-Yu, C. y Llopart, M. (2019). Sustaining students' cultures and identities. A qualitative study based on the Funds of knowledge and identity approaches. Sustainability, 11(12), 1-12. https://doi.org/10.3390/ su11123400.

Garcia, A. (2017). Privilege, power, and Dungeons \& Dragons: How systems shape racial and gender identities in tabletop role-playing games. Mind, Culture, and Activity, 24(3), 232-246. https://doi.org/10.1080/10749039.2017.1293691.

Gee, J. P. (2004). Situated Language and Learning: A Critique of Traditional Schooling. New York, NY: Routledge.

Gee, J. P. (2010). New Digital Media and Learning as an Emerging Area and "Worked Examples" As One Way Forward. Cambridge, MA: The MIT Press.

Gee, J. P., y Esteban-Guitart, M. (2019). Designing for deep learning in the context of digital and social media. Comunicar, 58, 9-17. https://doi.org/10.3916/C58-201901.

González-Martínez, J., Serrat-Sellabona, E., Estebanell-Minguell, E., Rostan-Sánchez, C. y Esteban-Guitart, M. (2018). Sobre el concepto de alfabetización transmedia en el ámbito educativa. Una revisión de la literatura. Comunicación y Sociedad, 33, 15-40. http://dx.doi.org/10.32870/cys.v0i33.7029.

González-Patiño, J. y Esteban-Guitart, M. (2014). Some of the challenges and experiences of formal education in the Mobile-Centric Society. Digital Education Review, 25, 64-86. https://doi.org/10.1344/der.2014.25.64-86.

Gutiérrez, K. y Rogoff, B. (2003). Cultural ways of learning: Individual traits or repertoires of practice. Educational Researcher, 32(5), 19-25. https://doi. org/10.3102/0013189X032005019.

Harackiewicz, J. M., Smith, J. L. y Prinski, S. J. (2016). Interest matters: The importance of promoting interest in education. Policy Insights from the Behavioral and Brain Sciences, 3(2), 220-227. https://doi.org/10.1177/2372732216655542.

Hidi, S. y Renninger, K. A. (2006). The four-phase model of interest development. Educational Psychologist, 41(2), 111-127. https://doi.org/10.1207/s15326985ep4102_4.

Hernández-Hernández, F., Sancho-Gil, J. M. y Domingo-Coscollola, M. (2018). Cartographies as spaces of inquiry to explore of teachers' nomadic learning trajectories. Digital Education Review, 33, 105-119. https://doi.org/10.1344/ der.2018.33.105-119.

Holzkamp, K. (2013). Psychology: Social self-understanding on the reasons for action in the conduct of everyday life. En E. Schraube y U. Ostkerkamp (Eds.), Psychology from the Standpoint of the Subject: Selected Writings of Klaus Holzkamp (pp. 233341). Basingstoke, UK: Palgrave Macmillan. 
Ito, M., Baumer, S., Bittanti, M., Boyd, D., Cody, R., Herr-Stephenson, B., ... Tripp, L. (2009). Hanging Out, Messing Around, and Geeking Out. Kids Living and Learning with New Media. Cambridge, MA: The MIT Press.

Ito, M., Gutiérrez, K., Livingstone, S., Penuel, W., Rhodes, J., Salen, K., ... Watkins, C. (2013). Connected Learning: An Agenda for Research and Design. Irvine, CA: Digital Media and Learning Research Hub.

Ito, M., Martin, C., Pfister, R. C., Rafalow, M. H., Salen, K. y Wortman, A. (2019). Affinity Online: How Connection and Shared Interest Fuel Learning. New York, NY: New York University Press.

Jenkins, H. (1992). Textual Proachers: Television Fans \& Participatory Culture. New York, NY: Routledge.

Jenkins, H. (2006). Convergence Culture. Where Old and New Media Collide. New York, NY: New York University Press.

Jenkins, H. (2009). Confronting the Challenges of Participatory Culture: Media Education for the 21st Century. Cambridge, MA: The MIT Press.

Jenkins, H., Al Shafei, E. y Gee, J. (2018). Do we still believe that networked youth can change the world? Special issue. Papeles de Trabajo sobre Cultura, Educación y Desarrollo Humano, 14(3), 1-51.

Jenkins, H., Shresthova, S., Gamber-Thompson, L., Kligler-Vilenchik, N. y Zimmerman, A. M. (2016). By Any Media Necessary: The New Youth Activism. New York, NY: New York University Press.

Jurow, A. S., Shea, M. y Van Steenis, E. (2016). Extending the consequentiality of "invisible work" in the food justice movement. Cognition and Instruction, 34(3), 210-221. https://doi.org/10.1080/07370008.2016.1172833.

Kumpulainen, K., Kajamaa, A. y Rajala, A. (2018). Understanding educational change: Agency-structure dynamics in a novel design and making environment. Digital Education Review, 33, 26-38. https://doi.org/10.1344/der.2018.33.26-38.

Martin, K. (2018). Supporting youth to envision careers in computer science. En W. Tierney, Z. Corwin y A. Ochsner (Eds.), Diversifying Digital Learning: Online literacy and educational opportunity (pp. 126-150). Maryland, USA: John Hopkins University Press.

O’Byrne, W. I., Schenke, K., Willis, J. E. y Hickey, D. T. (2015). Digital badges: Recognizing, assessing, and motivating learners in and out of school contexts. Journal of Adolescent and Adult Literacy, 58(6), 451-454. https://doi.org/10.1002/jaal.381.

Paris, D. (2012). Culturally sustaining pedagogy: A needed change in stance, terminology, and practice. Educational Researcher, 41(3), 93-97. https://doi. org/10.3102/0013189X12441244.

Penuel, W. R. (2006). Implementation and effects of 1:1 computing initiatives: A research synthesis. Journal of Research on Technology in Education, 38(3), 329-348. https://doi.org/10.1080/15391523.2006.10782463. 
Penuel, W. R. y DiGiacomo, D. K. (2017). Connected learning. En K. Peppler (Ed.), The Sage Encyclopedia of Out-of-School Learning (pp. 132-136). Londres, UK: SAGE.

Penuel, W. R. y DiGiacomo, D. K. (2018). Organizing learning environments for relational equity with new digital media. En P. Resta, y T. Laferrière, (Eds.), International Handbook of Information Technology in Primary and Secondary Education, 2nd Edition (pp. 943-949). Singapore: Springer.

Penuel, W. R. y O'Connor, K. (2018). From designing to organizing new social futures: Multiliteracies pedagogies for today. Theory into Practice, 57(1), 64-71. https://doi. org/10.1080/00405841.2017.1411715.

Penuel, W. R., Clark, T. L. y Bevan, B. (2016). Infrastructures to support equitable STEM learning across settings. Afterschool Matters, 24, 12-20.

Penuel, W. R., Chang-Order, J. y Michalchik, V. (2018). Using research-practice partnership to support interest-related learning in libraries. En V. R. Lee y A. L. Phillips (Eds.), Reconceptualizing Libraries. Perspectives from the Information and Learning Sciences (pp. 239-256). New York, NY: Routledge.

Penuel, W. R., DiGiacomo, D. K., Van Horne, K. y Kirshner, B. (2016). A Social Practice Theory of Learning and Becoming across Contexts and Time. Frontline Learning Research, 4(4), 30-38.

Pinkard, N. y Austin, K. (2011). Digital Youth Network: Creating New Media Citizens Through the Affinity Learning Model. International Journal of Learning and Media, 2(4). https://doi.org/10.1162/ijlm_a_00055.

Renninger, K. A. y Hidi, S. (2016). The Power of Interest for Motivation and Engagement. New York, NY: Routledge.

Rogoff, B. (2016). Culture and participation: a paradigm shift. Current Opinion in Psychology, 8, 182-189. https://doi.org/10.1016/j.copsyc.2015.12.002.

Subero, D., Vujasinovic, E. y Esteban-Guitart, M. (2017). Mobilising funds of identity in and out of school. Cambridge Journal of Education, 47(2), 247-263. https://doi. org/10.1080/0305764X.2016.1148116.

Subero, D., Llopart, M., Siqués, C. y Esteban-Guitart, M. (2018). The mediation of teaching and learning processes through identity artefacts. A Vygotskian perspective. Oxford Review of Education, 44(2), 156-170. https://doi.org/10.1080/03054985. 2017.1352501.

Vygotsky, L. S. (1963). Learning and mental development at school age. En B. Simon, y J. Simon (Eds.), Educational psychology in the U.S.S.R. (pp. 21-34). London, UK: Routledge \& Kegan Paul.

Vygotsky, L. S. (1997). Educational psychology. Boca Raton, FL: St. Lucie Press.

VV.AA. (2014). Learning Labs in Libraries and Museums: Transformative Spaces for Teens. Washington, DC: Association of Science-Technology Centers. 